\title{
L Shaped via based Mushroom type High Impedance Structure
}

\author{
Touseef Ahmad \\ Universitivy of Engineering and Technology Peshawar, Pakistan \\ touseefkhattak2013@gmail.com \\ Received: 27 March, Revised: 03 April, Accepted: 08 April
}

\begin{abstract}
High-impedance Electromagnetic Band-Gap structures (EBG) surfaces have the capability to forbid flow of EM waves in a given band which and therefore surface waves in case of planar antennas like mictostrip antenna can be minimized with this characteristics of EBG plane. Shape, size, symmetry, and material used in their construction defines their operating band. In this research, a novel compact EBG structure also called high impedance structure (HIS) is proposed. The design is achieved through incorporation of ' $\mathrm{L}$ ' shaped via to conventional mushroom type EBG/HIS instead of straight vias. The design includes distribution of square patches over substrate material below which there exists a ground plane. Vias passing through the substrate connecting square patches and the ground plane are also part of its design It has been observed that operating frequency of $\mathrm{L}$ shaped via based EBG is much lower than that of conventional mushroom type EBG/HIS having straight vias. Alternatively, we can say that size reduction has been achieved through incorporation of $\mathrm{L}$ shaped via to the EBG/HIS resulting in $62.5 \%$ of size reduction. All the designs and simulations are carried out in CST microwave studio.
\end{abstract}

Keywords - Microstrip patch antenna, high impedance surface, substrate, bandwidth.

\section{INTRODUCTION}

One of emerging materials that has been engineered artificially is metamaterial. By definition these surfaces satisfy two conditions, i.e they are purely artificial and do not exist naturally, and secondly EM properties possessed by these structures is superior and different from their parent elements [1]. EM and optical characteristic offered by these artificial materials are very much unique as well as different from their constituents. This phenomenon is achieved through inclusion, discontinuities and pattern of host material due to which their EM fields interact differently resulting in modification of EM properties like of host materials permittivity $(\varepsilon)$, permeability $(\mu)$, refractive index (n) etc of constituent materials. As these materials provides control over EM properties therefore some desired electromagnetic and optical performance can be achieved through them.

Since 1990s, rigorous analysis has been carried out for Single Negative (SNG) [2], Double Negative (DNG) metamaterials [3, 4] and planar Electromagnetic Band Gap
(EBG) structures [5]. Sievenpiper in [6] introduced 2D mushroom type EBG surfaces that were structured through array of unit cells distributed in periodic manner. This unit cell is composed of square patch placed over dielectric substrate below which there exist ground plane. A conducting cylindrical pin connects the square patch with ground plane which is passing through the substrate hence forming mushroom like configuration. These periodic structures possess the ability of suppression of propagation of surface energy waves along with in-phase reflection in a given frequency band. In-phase reflection characteristics enable these structures to have image currents and antenna current both with same phase that results in constructive interference resulting in better antenna performance. If we look into the structural configuration of high impedance structure, we will see that its composition incudes two-dimensional lattice of resonating elements that form basic two dimensional stop band filter to forbid flow of surface currents.

As explained, high-impedance Electromagnetic Band-Gap structures (EBG) surfaces have the capability to forbid flow of EM waves in a given band which is another important feature. Surface waves in case of planar antennas like mictostrip antenna can be minimized with the help of EM wave suppression characteristics of EBG plane. An initial research on EBG structure at microwave frequencies was conducted by Prof. E. Yablanovitch and his research group in [7], which explained many of its properties (surface current suppression and zero reflection phase) using an effective surface impedance model. Since then, theory and practical applications of EBG structure has become an extensive research area due to its unusual properties and design flexibilities. Consequently, numerous of EBG structures have been successfully employed to realize novel high performance devices such as filters, waveguides, antennas, etc. [8], [9], [10].

Low profile wire antennas with EBG ground are studied in [11], [12], [13]. In [14] and [15], EBG structure is employed to on-body antennas to reduce sensitive absorption rate (SAR). Other applications of EBG structures such as Multi-band antenna, LTCC and bandwidth enhancement are introduced in [16], [17] and [18], respectively.

Another area of EBG structure research is focused on characteristics of its unique properties, providing simple and fast design approaches. [19] presents a study on in-phase reflection and wave suppression characteristics of EBG 
structure. In [20], a method of designing controlled bandwidth EBG structure is demonstrated. Spectral domain method is explained in [21] to prove that capacitive surfaces can perform perfect magnetic conductors in a relative low frequency range. Requirement for miniaturization exists in every design whether it is related to antenna, amplifiers, filters or some other RF components. Therefore, EBG structure with compact size is required in several applications. Several attempts have been carried out in different articles [22-24]. Edge located via based mushroom type EBG was presented in [25] for demonstrating reduction in size of the EBG. Another design introduced in [26] used helical shaped via in their design for size compactness of EBG plane.

In this research, a novel compact EBG structure is proposed. The design is achieved through incorporation of ' $\mathrm{L}$ ' shaped via to conventional mushroom type EBG instead of straight vias. The design includes distribution of square patches over substrate material below which there exists a ground plane. Vias passing through the substrate connecting square patches and the ground plane are also part of its design. Geometrical shape of via can vary response of the EBG. In our case straight via is modified and transformed into $L$ shaped via. This modification is carried out by moving both ends of straight via over square patches in opposite direction. Band gap response and parametric study of the newly designed $L$ shaped based EBG is studied. A comparison between conventional mushroom type EBG \& $\mathrm{L}$ shaped EBG is also carried out in order to analyze the benefit of L shaped EBG.

Sequence of the paper is as following. Section II mainly deals design parameters of HIS. Section III presents design of L shaped via base mushroom type HIS alongwith discussion of results. At the end conclusion of the research work is presented.

\section{HIGH IMPEDANCE SURFACE}

Square patch EBG structures is the conventional design which is also termed as high impedance structures (HIS) that was proposed by Sievenpiper [27-31] which finds application in variety of low profile efficient antennas. Rahmat Samii [32] describes EBG as a periodic or a periodic configuration of multi dimension having power to stop or enhance the transmission of electromagnetic waves in a certain frequency range with no conditional limit of incident angel and polarization. Bandgap is the frequency range in which the surface waves are suppressed. Substrate thickness and dimensions of the EBG unit-cell affect the band gap characteristic. The unit cell may be either square or rectangular in shape. The mushroom, loop, or spiral may be the resonating element. Rahmat-Samii [33] and Sievenpiper [34] designed several kinds of EBG and HIS configuration which were further used and find its application in a variety of low profile linear and circularly polarized antennas. HIS design by Sievenpiper is shown below in Figure 1 and 2.

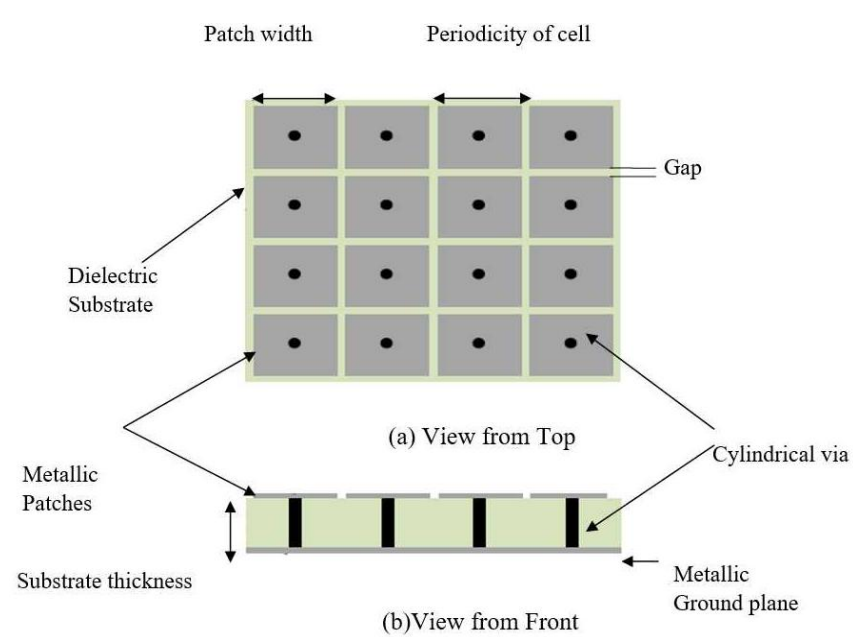

Figure 1. High impedance Surface (Sievenpiper) (a) Top-View (b) FrontView

Planar structures give in-phase reflection and suppress surface propagation in a particular frequency band. Additionally, the antenna currents are in-phase with the image currents, therefore both constructively interfere and provide better antenna performance[35].

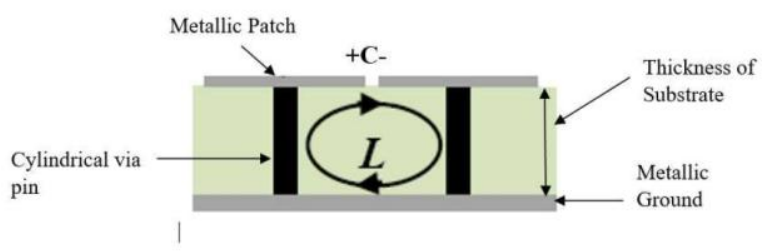

(a)

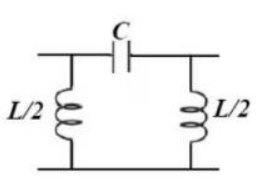

(b)

Figure 2. Equivalent circuit (LC) model (a) Front-View (b) Equivalent resonant circuit (LC)

To explain operation of HIS, consider Figure 4. It can be seen that HIS behavior is just parallel resonant circuit. The charge distribution among neighboring cells correspondent to capacitance $\mathrm{C}$ while the current flow through metallic via from top and bottom results to inductance $\mathrm{L}$.

Following formula determines $\mathrm{L}$ and $\mathrm{C}$ values [33].

$$
\begin{gathered}
C=\frac{W\left(\varepsilon_{0}+\varepsilon_{r}\right)}{\pi} \cosh ^{-1}\left(\frac{2 W+g}{g}\right) \\
L=\mu h
\end{gathered}
$$

Here

$\mathrm{W}=$ Width of the patch

$\mathrm{g}=$ Gap between two adjacent patches 
$\varepsilon_{\mathrm{r}}=\mathrm{EBG}$ substrate relative permittivity

$\mathrm{h}=$ Thickness of the substrate used

$\mu=$ Medium permeability

Following equation expressed resonant frequency $\mathrm{f}_{\mathrm{r}}$

$$
f_{r}=\frac{1}{2 \pi \sqrt{L C}}
$$

From above equation the Frequency Band Gap (BW) can be come near to as

$$
\mathrm{BW}=\frac{\Delta \omega_{0}}{\omega_{0}}=\frac{1}{\mathbf{n}} \sqrt{\frac{\mathrm{L}}{\mathrm{C}}}
$$

Here $\boldsymbol{\eta}$ is the intrinsic impedance of free-space $=120 \pi$

By suppressing surface waves, these assemblies are very co-operative just in case of planer antennas resulting in directivity, efficiency of the antenna and achieving high gain [36-37]. EBGs can also be utilized for GPS applications [3839]. It has been observed that when EM wave strike Perfect Electric Conductor or planner EBGs, sense of polarization reverses resulting in mismatching in polarization. In order to side-step this kind of mismatch, PDEBGs (Polarization dependent EBGs) in several applications are introduced [4041].

\section{RESULTS AND DISCUSSIONS}

This section represents the design of mushroom type EBG with $\mathrm{L}$ shaped vias. The design includes distribution of square patches over substrate material below which there exists a ground plane. Vias passing through the substrate connecting square patches and the ground plane are also part of its design. Geometrical shape of via can vary response of the EBG. In our case straight via is modified and transformed into $\mathrm{L}$ shaped via. This modification is carried out by moving both ends of straight via over square patches in opposite direction. Figure 3 shows this transformation.

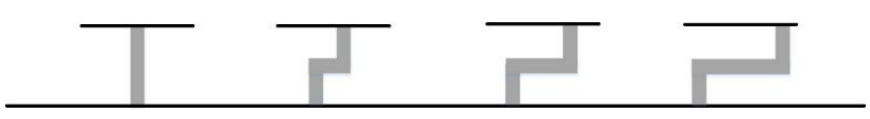

Figure 3. Transformation of Straight Vias to L shaped Vias

Structural configuration of designed mushroom type EBG with $\mathrm{L}$ shaped vias is given in figure 4.

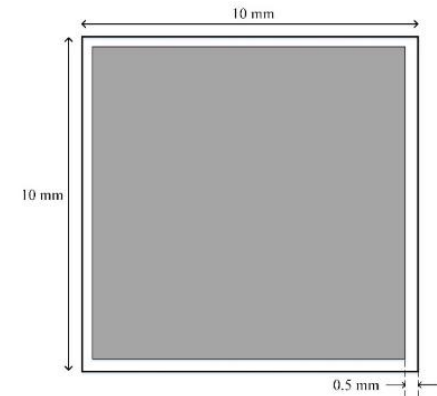

(a)

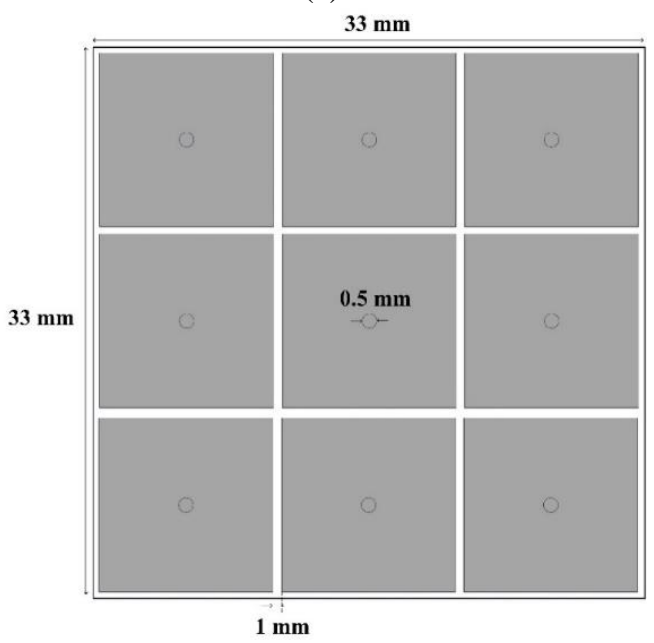

(b)

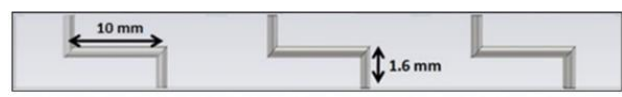

(c)

Figure 4. Mushroom type EBG with L shaped Vias (a) Unit Cell Model (b) Top view of 3x3 EBG plane (c) Side View of 3x3 EBG plane

Here FR-4 substrate is used for the design of EBG having dielectric constant of 4.3 with thickness of $3.2 \mathrm{~mm}$. Dimension of square patch is $10 \mathrm{~mm} \times 10 \mathrm{~mm}$ and a total of nine patches are taken for the design. Gap between square patches is $1 \mathrm{~mm}$ and radius of connecting vias is taken to be $0.25 \mathrm{~mm}$. Overall dimension of $3 \times 3$ patch mushroom type EBG is $33 \mathrm{~mm} \times 33$ $\mathrm{mm}$.

To evaluate band gap behavior of the EBG, suspended microstrip line technique is utilized. For this purpose, a microstrip line is placed over the EBG ground plane and excited on both of its port thus forming a to port network. Coupling between the two port of transmission line i.e S21or $\mathrm{S} 12$ will show the band gap response of the EBG. As surface impedance of EBG plane is very high, therefore propagation of EM waves will be blocked in side band gap and similarly EM wave transmission will be high in rest of the band. Hence reduction if S21 will be observed within bandgap.

To analyzer bandgap response of the mushroom type EBG with $\mathrm{L}$ shaped, a microstrip line having length of $33 \mathrm{~mm}$ and width of $2 \mathrm{~mm}$ is placed over $3 \times 3$ patch mushroom type EBG with $\mathrm{L}$ shaped vias and excited. The configuration of design is given in figure 5 . 


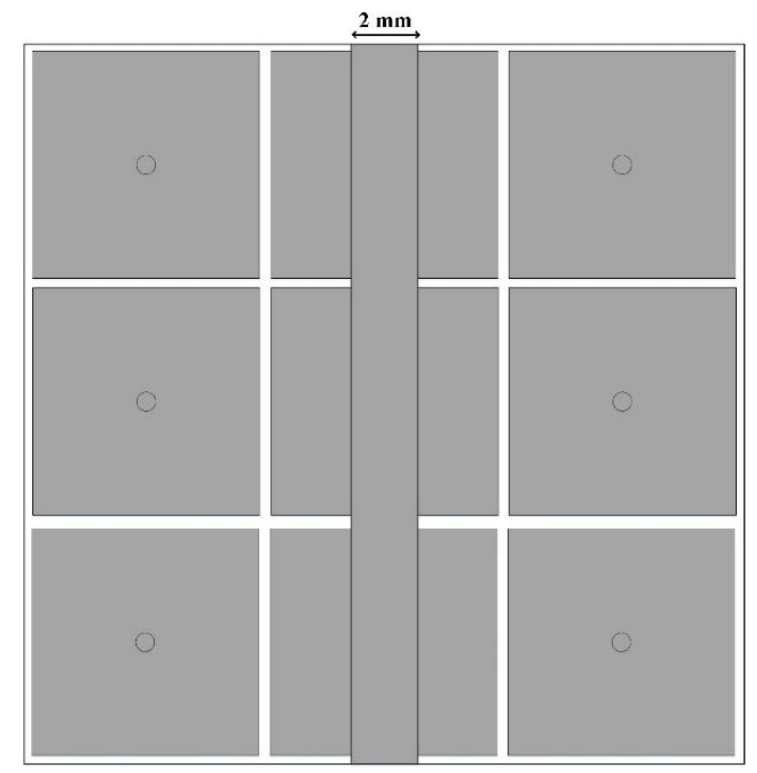

(a)

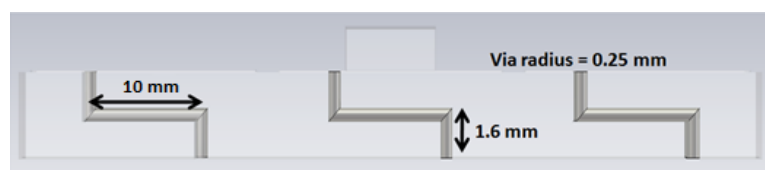

(b)

Figure 5. Suspended transmission line over Mushroom type EBG with L shaped Vias (a) Top View (b) Side View

Frequencies, having S21 less than $-10 \mathrm{~dB}$, are defined as the band gap normally. Simulated S21 of mushroom type EBG with $\mathrm{L}$ shaped vias is presented in figure 6 . This can be seen that value of $\mathrm{S} 21$ decreases to $-40 \mathrm{~dB}$ near $1.3 \mathrm{GHz}$ having -10 $\mathrm{dB}$ stop band of $112 \mathrm{MHz}$ ranging from $1.253 \mathrm{GHz}$ to 1.366 $\mathrm{GHz}$.

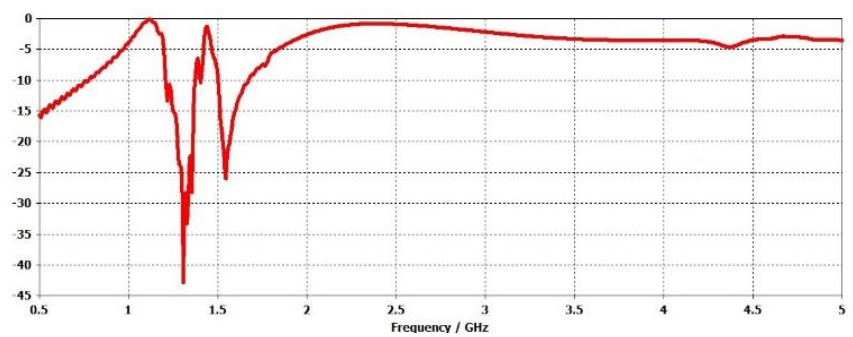

Figure 6. S21/Band gap for Mushroom Type EBG with L shaped Vias

Afterwards conventional mushroom type EBG having straight vias is also designed. In this case all the parameters are kept same as that of mushroom type HIS with L shaped via except configuration of the via. It was observed that its operating frequency band is centered at $2.1135 \mathrm{GHz}$ having -10 $\mathrm{dB}$ stop band of $310 \mathrm{MHz}$ ranging from $1.9559 \mathrm{GHz}$ to 2.2659 $\mathrm{GHz}$ as depicted in figure 7 .

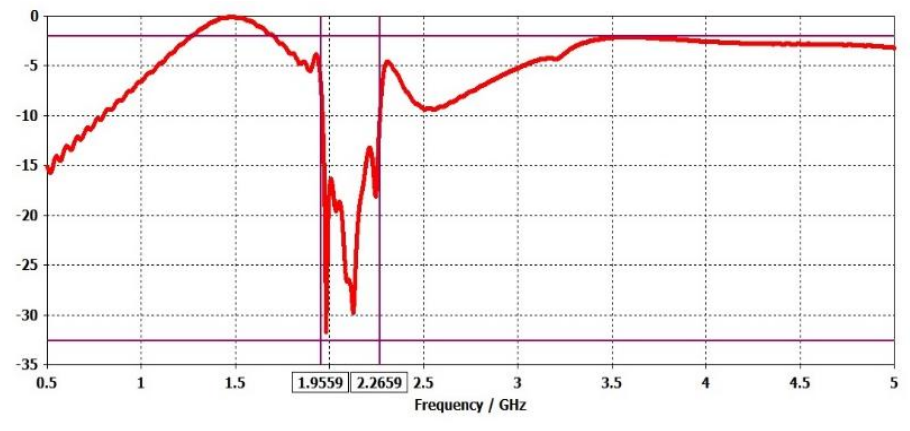

Figure 7. S21/Band gap for Mushroom Type EBG with Straight Vias

From these results we can clearly see that operating frequency of $\mathrm{L}$ shaped via based EBG is much lower than that of conventional mushroom type EBG. Alternatively, we can say that size reduction has been achieved through incorporation of $\mathrm{L}$ shaped via to the EBG resulting in $62.5 \%$ of size reduction.

\section{CONCUSLION}

This article discusses miniaturization of conventional mushroom type EBG/HIS. Desired response is achieved through modification of via configuration of HIS. For this puspose the straight vias of HIS plane is replaced with $\mathrm{L}$ shaped vias. It has been observed that band gap of conventional mushroom type HIS with straight via is centred at $2.1135 \mathrm{GHz}$ having $-10 \mathrm{~dB}$ stop band of $310 \mathrm{MHz}$ ranging from 1.9559 $\mathrm{GHz}$ to $2.2659 \mathrm{GHz}$. Wheras band gap of mushroom type HIS with $\mathrm{L}$ shaped vias was centred at $1.3 \mathrm{GHz}$ having $-10 \mathrm{~dB}$ stop band of $112 \mathrm{MHz}$ ranging from $1.253 \mathrm{GHz}$ to $1.366 \mathrm{GHz}$. Hence $62.5 \%$ reduction is operating band of mushroom type HIS was observed through incorporation of $L$ shaped vias i.e $62.5 \%$ size reduction is achieved.

\section{REFERENCES}

[1] A.Sihvola,-Electromagnetic emergence in metamaterials\|, Advances in Electromagnetics of Complex Media and Metamaterials, vol. 89, pp. 1$17,2003$.

[2] J. Zehentner and J. Machac, -Volumetric single negative metamaterials\|, Proceedings of Metamaterials Congress, pp. 22-24, 2007

[3] N. Engheta,-Metamaterials with negative permittivity and permeability: background, salient features, and new trends\|, IEEE MTTS International Microwave Symposium Digest, pp. 187-190, 2003.

[4] N. Engheta,-Design, fabrication, and testing of double negative metamaterialsl, IEEE Transactions on Antennas and Propagation, vol. 51, issue. 7, pp. 1516-1529, 2003.

[5] W. R. Ziolkowski and N. Engheta, -Metamaterials: Physics and Engineering Explorationsl, John Wiley \& Sons, Inc., 18 September 2006.

[6] D. Sievenpiper, -High Impedance Electromagnetic Surfaces\|, Ph.D. dissertation, Electrical Engineering Department, University of California, Los Angeles, 1999.

[7] D. Sievenpiper, L. Zhang, R. F. J. Broas, N. G. Alexopolous, E. Yablonovitch,, "High-Impedance Electromagnetic Surfaces with a Forbidden Frequency Band," IEEE Transactions on Microwave Theory and Techniques, vol. 47, no. 11, pp. 2059-2074, 1999.

[8] Y. J. Lee, J. Yeo, R. Mittra, W. S. Park, "Application of Electromagnetic Bandgap (EBG) Superstrates With Controllable Defects for a Class of Patch Antennas as Spatial Angular Filters," IEEE 
Transactions on Antennas and Propagation, vol. 53, no. 1, pp. 224-235, 2005.

[9] S. G. Mao, M. Y. Chen, "Propagation Characteristics of Finite-Width Conductor-Backed Coplanar Waveguides with Periodic Electromagnetic Bandgap Cells," IEEE Transactions on Microwave Theory and Techniques, vol. 50, no. 11, pp. 2691-2703, 2002.

[10] F. Yang, Y. Rahmat-Samii, "Reflection Phase Characterizations of the EBG Ground Plane for Low Profile Wire Antenna Applications," IEEE Transactions on Antennas and Propagation, vol. 51, no. 10, pp. 677-682, 2003.

[11] F. Yang, Y. Rahmat-Samii, "A low-profile circularly polarized curl antenna over an electromagnetic bandgap (EBG) surface," Microwave and Optical Technology Letters, vol. 31, no. 4, pp. 264-267, 2001.

[12] Z. Li, Y. Rahmat-Samii, "PBG, PMC and PEC ground planes: A case study of dipole antennas," in IEEE Antennas and Propagation Society International Symposium, 2000.

[13] T. H. Liu, W. X. Zhang, M. Zhang, K. F. Tsang, "Low profile spiral antenna with PBG substrate," Electronics Letters, vol. 36, p. 779-780, 2000.

[14] [14] S. Bashir, M. Hosseini, R. M. Edwards, M. I. Khattak, L. Ma, "Bicep Mounted Low Profile Wearable Antenna Based on A NonUniform EBG Ground Plane - Flexible 60 EBG Inverted-L (FEBGIL)," in Loughborough antennas and propagation conference, Loughborough, 2008

[15] N. Chahat, M. Zhadobov, R. Sauleau, K. Mahdjoubi, "Improvement of the On-Body Performance of a Dual-Band Textile Antenna Using an EBG structure," in Loughborough antennas and propagation conference, Loughborough, 2010.

[16] J. H. S. D. Sievenpiper, "Textured Surface Having High Electromagnetic Impedance in Multiple Frequency Bands". United States Patent US 6,483,481 B1, 19 November 2002.

[17] R. Li, G. DeJean, M. M. Tentzeris, J. Papapolymerou, J. Laskar, "Radiation-Pattern Improvement of Patch Antennas on a Large-Size Substrate Using a Compact Soft-Surface Structure and Its Realization on LTCC Multilayer Technology," IEEE Transactions on antennas and propagation, vol. 53, no. 1, pp. 200-208, 2005.

[18] R. Diaz, V. Sanchez, E. Caswell, A. Miller, "Magnetic Loading of Artificial Magnetic Conductors for Bandwidth Enhancement," 2003.

[19] Aminian, F. Yang, Y. Rahmat-Samii, "In-phase Reflection and EM Wave Suppression Characteristics of Electromagnetic Band Gap Ground Planes," IEEE Antennas and Propagation Society International Symposium," 2003.

[20] [20] Aminian, Y. Rahmat-Samii, "Bandwidth Determination for Soft and Hard Ground Planes: A Unified Approach in Visible and Surface Wave Regions," in IEEE Antennas and Propagation Society International Symposium, 2004.

[21] [21] F. Bilotti, L. Vegni, "Radiating Features of Capacitive and Inductive Surfaces," Microwave and Optical Technology Letters, vol. 39, no. 2, pp. 117-121, 2003.

[22] Stylianos D. Assimonis, Traianos V. Yioultsis, and Christos S. Antonopoulos, "Design and Optimization of Uniplanar EBG Structures for Low Profile Antenna Applications and Mutual Coupling Reduction," IEEE Transaction on antennas and propagation, vol. 60, no. 10, Oct. 2012, pp. 4944-4949.

[23] S. Zhang, B. Kiong Lau, Y. Tan, Z. Ying, and S. He, "Mutual Coupling Reduction of two PIFAs with a T-Shape Slot Impedance Transformer for MIMO Mobile Terminals," IEEE Transaction on antennas and propagation, vol. 60, no. 3, March 2012, pp. 1521-1531.

[24] A. M. Abdelreheem, and M. A. Abdalla, "A Novel Bilateral UC-EBG Structure," IEEE Antenna and Propagation Society International Symposium pp. 1780-81, July 2014, pp. 1780-1781.

[25] Eva Rajo-Iglesias, Member, IEEE, Luis Inclán-Sánchez, José-Luis Vázquez-Roy, Member, IEEE, and Enrique García-Muñoz, "Size Reduction of Mushroom-Type EBG Surfaces by Using Edge-Located Vias" IEEE MICROWAVE AND WIRELESS COMPONENTS LETTERS, VOL. 17, NO. 9, SEPTEMBER 2007.

[26] Wenquan Cao Bangning Zhang Tongbin Yu Daosheng Guo Aijun Liu "Helical-Via-Type Mushroom EBG Structure for Size
Reduction" IEEE Antenna and Propagation Society International Symposium pp. 1780-81, June 2015, pp. 1347-1349

[27] D. Sievenpiper, -High-Impedance Electromagnetic Surfaces with a Forbidden Frequency Bandll, IEEE Transactions on Microwave Theory and Techniques, vol. 47, pp. 2059-2073, November 1999.

[28] D. Sievenpiper, L. Zhang and E. Yablonovitch, -High-Impedance Electromagnetic Ground Planes\|, IEEE MIT-S Digest, vol. 4, pp. 1529. 1532,1999

[29] D. Sievenpiper, R. Broas and E. Yablonovitch, -Antennas on highimpedance ground planesl, IEEE MTT-S, International Microwave Symposium Digest, vol. 3, pp. 1245-1248, 1999.

[30] D. Sievenpiper, - High Impedance Electromagnetic Surfaces, Ph.D. dissertation, Electrical Engineering Department, University of California, Los Angeles, 1999

[31] F. Yang, Y. Rahmat-Samii, -Electromagnetic Band Gap Structures in Antenna Engineering\|, The Cambridge RF and Microwave Engineering Series, October 2008.

[32] B. Jecko, T. Monediere, L. Leger, "High Gain EBG Resonator Antenna", 18th International Conference on Applied Electromagnetics and Communications, ICECom 2005, pp. 1-3, 12-14 Oct. 2005

[33] D. Sievenpiper, L. Zhang and E. Yablonovitch, "High-Impedance Electromagnetic Ground Planes", IEEE MIT-S Digest, vol. 4, pp. 1529 1532, 1999

[34] Qian Y., Coccioli R., Sievenpiper D., Radisic V., Yablonovitch E., and Itoh T., "A Microstrip Patch Antenna using novel photonic bandgap structures", Microwave J., vol 42, pp. 66-76, Jan 1999.

[35] Z. Duan, D. Linton, W. Scanlon, and G. Conway, "Using EBG to Improve Antenna Efficiency in Proximity to the Human Body", Institution of Engineering and Technology Seminar on Wideband, Multiband Antennas and Arrays for Defence or Civil Applications, pp. 173-180, London, 13-13 March 2008.

[36] X. L. Bao, G. Ruvio, M. J. Ammann, and M. John, "A novel GPS patch antenna on fractal Hi-Impedance surface Substrate", IEEE Antenna and Wireless Propagation Letters, vol. 5, 2006

[37] R. Baggen, M. Martínez-Vázquez and J. Leiss, "Low Profile GALILEO Antenna using EBG Technology", IEEE Transactions on Antennas and Propagation, vol. 56, no. 3, pp. 667-674, March 2008.

[38] F. Yang, Y. Rahmat-Samii, "Polarization-Dependent Electromagnetic Band Gap (PDEBG) structures: designs and applications", Microwave and Optical Technology Letters, vol. 41, issue. 6, pp. 439-444, June 20, 2004.

[39] Y. Fu and N. Yuan, "Surface-wave bandgap of Polarisation dependent Electromagnetic bandgap Structures", Microwave and Optical Technology Letters, vol. 49, issue 4, pp. 946-949, 26 February 2007.

[40] D. Yan, Q. Gao, C. Wang, C. Zhu, N. Yuan, "A novel polarisation convert surface based on artificial magnetic conductor", Asia-Pacific Microwave Conference Proceedings, APMC 2005, vol. 3, pp. 4-7, December 2005

[41] P. J. Ferrer, B. Kelem and C. Craeye, "Design of broadband transpolarizing surfaces", Microwave and Optical Technology Letters, vol. 48, no. 12, pp. 2606-2611, December 2006. 